\title{
gु \\ Localization of the phantom force induced by the tunneling current
}

\author{
Thorsten Wutscher, Alfred J. Weymouth, ${ }^{*}$ and Franz J. Giessibl \\ Institute of Experimental and Applied Physics, University of Regensburg, 93040 Regensburg, Germany
}

(Received 9 March 2012; published 14 May 2012)

\begin{abstract}
The phantom force is an apparently repulsive force, which can dominate the atomic contrast of an AFM image when a tunneling current is present. We described this effect with a simple resistive model, in which the tunneling current causes a voltage drop at the sample area underneath the probe tip. Because tunneling is a highly local process, the areal current density is quite high, which leads to an appreciable local voltage drop that in turn changes the electrostatic attraction between tip and sample. However, $\mathrm{Si}(111)-7 \times 7$ has a metallic surface state and it might be proposed that electrons should instead propagate along the surface state, as through a thin metal film on a semiconducting surface, before propagating into the bulk. In this paper, we first measure the phantom force on a sample that displays a metallic surface state [here, $\mathrm{Si}(111)-7 \times 7]$ using tips with various radii. If the metallic surface state would lead to a constant electrostatic potential on the surface, we would expect a direct dependence of the phantom force with tip radius. In a second set of experiments, we study $\mathrm{H} / \mathrm{Si}(100)$, a surface that does not have a metallic surface state. We conclude that a metallic surface state does not suppress the phantom force, but that the local resistance $R_{S}$ has a strong effect on the magnitude of the phantom
\end{abstract} force.

DOI: 10.1103/PhysRevB.85.195426

PACS number(s): 68.37.Ps, 73.50.-h, 73.63.Rt

\section{INTRODUCTION}

Scanning probe microscopy (SPM) offers the possibility to determine structural and electronic properties of a surface on the atomic level. ${ }^{1,2}$ The two most common SPM techniques are scanning tunneling microscopy (STM) and frequency-modulation atomic force microscopy (FM-AFM). With combined STM and FM-AFM, we recently observed a so-called phantom force on $\operatorname{Si}(111)-7 \times 7 .{ }^{3}$ When the tip is too far from the surface to allow the resolution of chemical contrast in the force channel, atomic contrast can still be observed in constant-height mode with an applied bias. The resulting images appeared similar to the tunneling current images. In our proposed model, the tunneling current is injected from the tip into a small area within a radius of $\approx 100 \mathrm{pm}$, the approximate atomic radius of $\mathrm{Si}$. This causes an appreciable voltage drop that decreases the electrostatic attraction between tip and sample as a function of tunneling current, causing these phantom force images. However, a highly localized voltage drop leading to the phantom force effect appears to be incompatible with the existence of the metallic surface state of the $\operatorname{Si}(111)-7 \times 7$ surface.

The $\operatorname{Si}(111)-7 \times 7$ surface is described by the dimer-adatomstacking-fault (DAS) model. ${ }^{4}$ One unit cell of the surface consists of 12 adatoms, which have partially filled dangling bonds, forming a metallic surface state. ${ }^{5}$ An intriguing question is how electrons propagate through the metallic surface state, which is still under discussion and we refer the reader to Refs. 6-13. A popular description is that electrons, tunneling from a STM tip onto the surface, propagate along the metallic surface state before entering the bulk. To estimate the extension of a voltage drop on a surface with and without a metallic surface state, we used finite-element analysis (FEA) software to illustrate these two cases. ${ }^{14}$ For a surface without a metallic surface state, we modelled a silicon semiconductor sample with a constant conductivity, shown in Fig. 1(a). To mimic a surface with a metallic surface state, we added a thin metal sheet on top of the sample [Figs. 1(b)-1(d)]. The FEA was performed with increasing conductivities of the metal sheet, $\sigma_{s}=10^{2} \frac{\mathrm{s}}{\mathrm{m}}(\mathrm{b})$, $\sigma_{s}=10^{4} \frac{\mathrm{s}}{\mathrm{m}}(\mathrm{c})$, and $\sigma_{s}=10^{6} \frac{\mathrm{s}}{\mathrm{m}}(\mathrm{d})$, since these metal sheet conductivities cover the range of surface state conductivities noted in Ref. 10 , with, e.g., $10^{4} \frac{\mathrm{s}}{\mathrm{m}} \cdot 1 \AA$ corresponding to $1 \frac{\mu \mathrm{S}}{\square}$. In each case, (a)-(d), we defined a highly localized current source on the surface. The current density was set to $\frac{1 \mathrm{nA}}{(100 \mathrm{pm})^{2} \pi}=31 \frac{\mathrm{GA}}{\mathrm{m}^{2}}$. In Fig. 1(a), without a metal sheet, the voltage drop amounts to $230 \mathrm{mV}$ and is highly focused. In Figs. 1(b)-1(d), with a metal sheet, the voltage drop shows a reduction of its amount and an increasing lateral extension for increasing conductivities of the metal sheet. As we have observed the phantom force effect on the $\mathrm{Si}(111)-7 \times 7$ surface, the question remains how the metallic surface state relates to the phantom force.

This paper gives a description of the phantom force based on our model of an attractive electrostatic force in Sec. II. Section III introduces to the equipment and methods used for the experiments. In Sec. IV we investigate whether the metallic surface state has an effect on our observations on $\mathrm{Si}(111)-7 \times 7$. If it does, we would expect a delocalized effect and thus for the observed phantom force to depend on the macroscopic tip radius $R$, just as the electrostatic force between a plate and a semispherical tip depends on the radius. ${ }^{15,16}$ To evaluate this, we performed constant height images on $\mathrm{Si}(111)-7 \times 7$ to extract the ratio between the frequency shift due to the phantom force and the tunneling current ["phantom force slope," defined in Eq. (7)] and relate it to the macroscopic tip radius $R$, which was determined by force versus distance spectroscopy at zero effective bias. Following the conclusions from our finite-element analysis and our theory presented in Ref. 3, the phantom force is expected to occur on a surface without a metallic surface state. This has not yet been demonstrated experimentally. In Sec. V, we show the phantom force effect on a sample that does not have a metallic surface state. 
(a)

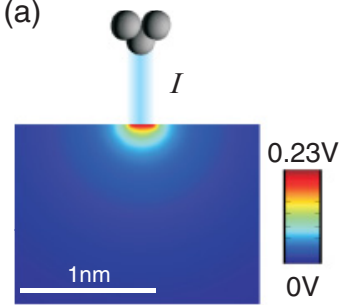

(c)
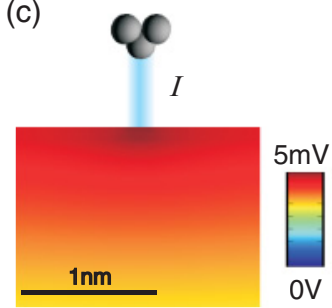

(b)

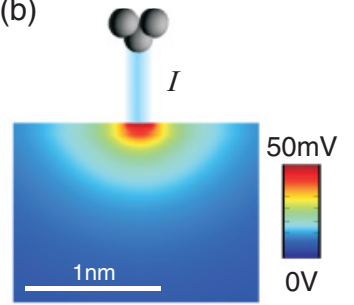

(d)

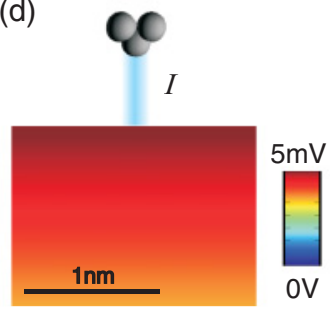

FIG. 1. (Color online) Finite-element analysis of a voltage drop in a plain bulk material (a) and a bulk material covered with a thin metal sheet on top (b)-(d) are shown. The bulk material has a constant conductivity of $\sigma_{b}=10 \frac{\mathrm{S}}{\mathrm{m}}$ [equal to the $\mathrm{Si}(111)$ sample used in the experiment]. In (b)-(d) the metallic surface state of $\mathrm{Si}(111)-7 \times 7$ is modelled by a metal sheet with a thickness of $100 \mathrm{pm}$. The conductivities of the metal sheet increase with $\sigma_{s}=10^{2} \frac{\mathrm{S}}{\mathrm{m}}$, (b), $\sigma_{s}=10^{4} \frac{\mathrm{s}}{\mathrm{m}}$, (c), to $\sigma_{s}=10^{6} \frac{\mathrm{s}}{\mathrm{m}}$ in (d). Without the conductive surface layer, (a), the voltage drop amounts to $230 \mathrm{mV}$ and is highly localized, while the conductive surface layer leads to a reduction and a lateral spreading of the voltage drop.

\section{THEORETICAL DESCRIPTION OF THE PHANTOM FORCE}

In this section, we introduce FM-AFM and describe the expected relation between the FM-AFM signal and the tunneling current in contrast to the relation between FM-AFM signal and tunneling current with a phantom force. Additionally, we mathematically derive the contribution of the tunneling current on the electrostatic force.

In FM-AFM, the forces between tip and sample cause a frequency shift $\Delta f$ relative to the resonance frequency $f_{0}$ of an unperturbated cantilever. ${ }^{17,18}$ The cantilever has a stiffness $k$ and oscillates with a constant amplitude $A$ at a distance $z$ from the surface. For small amplitudes, the relation between the force $F_{t s}$ and $\Delta f$ is $\Delta f \approx \frac{f_{0}}{2 k} k_{t s}$, where $k_{t s}=-\frac{d F_{t s}}{d z}$ is the force gradient between tip and sample. ${ }^{19}$ For a Morse potential, which describes the chemical interaction between tip and sample atom, the force and $\Delta f$ behave as shown in Fig. 2. In region I, as the tip approaches the sample, the force becomes more attractive and $\Delta f$ more negative. Approaching the tip closer to the sample, attractive chemical bonds start to form and $\Delta f$ decreases further. In region II, $\Delta f$ starts to increase. In this paper, we focus on region I, which is the region at the onset of current. A more detailed explanation of the behavior between force and $\Delta f$ is given, e.g., in Ref. 18 . When performing STM, the tunneling current exponentially increases with decreasing tip-sample distance..$^{20}$ If force and current were independent, we would expect a decrease in the frequency shift as the tunneling current increases on a $\Delta f$ versus $I$ plot. However, a surprising characteristic of the

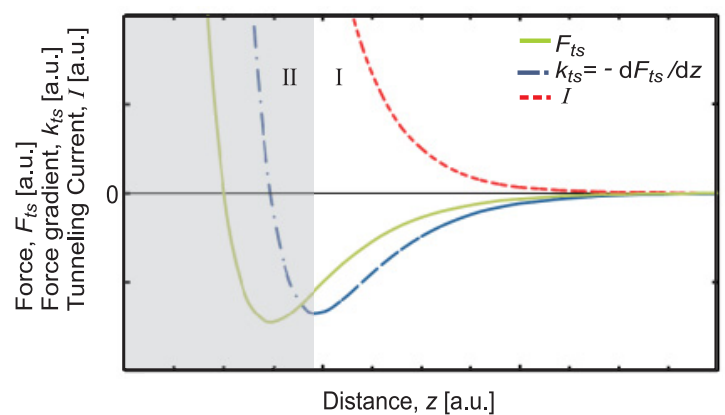

FIG. 2. (Color online) Qualitative distance dependence of the force $F_{t s}$ according to a Morse potential, the corresponding force gradient $k_{t s}$, and the tunneling current $I$. For small cantilever amplitudes, the frequency shift $\Delta f$ is proportional to $k_{t s}$. In region I, $\Delta f$ decreases, whereas $I$ increases. In region II, $\Delta f$ starts to increase.

phantom force is the increase of the frequency shift as the tunneling current increases when plotting $\Delta f$ against $I .^{3}$

The phantom force can be modelled by extending the formula of the attractive electrostatic force between two metal objects by a tunneling current dependent term. Without the influence of the tunneling current we can write

$$
F_{t s}^{e s}=-\frac{1}{2} \frac{d C_{t s}}{d z} V^{2}
$$

where $V$ is the potential difference between tip and sample, and $C_{t s}$ is the capacity of the tip-sample junction. If the tip was a flat surface $A$ at a distance $z$ to the sample, the derivative of capacity with distance would be given by

$$
\frac{d C_{t s}}{d z}=-\epsilon_{0} \frac{A}{z^{2}} .
$$

The permittivity of vacuum $\epsilon_{0} \approx 8.85 \mathrm{pF} / \mathrm{m}$ can also be expressed as $\epsilon_{0} \approx 8.85 \mathrm{pN} / \mathrm{V}^{2}$. Thus, for $A=20 z^{2}$, a force of about $90 \mathrm{pN}$ would arise for a bias of $1 \mathrm{~V}$, increasing with the square of voltage.

The effective bias responsible for the electrostatic force is $V=V_{\text {bias }}-V_{\mathrm{CPD}}$, where $V_{\text {bias }}$ is the applied voltage and $V_{\mathrm{CPD}}$ is the contact potential difference between tip and sample. ${ }^{21}$ While local changes in $V_{\mathrm{CPD}}$ (Ref. 22) will affect the local electrostatic attraction between tip and sample dependent upon $V_{\text {bias }}$, they cannot explain observations of this phantom force, for reasons discussed in Ref. 3: A local change in $V_{\mathrm{CPD}}$ would cause a $\Delta f$ decrease in one bias (assuming the applied $\left.\left|V_{\text {bias }}\right|>\left|V_{\mathrm{CPD}}\right|\right)$ and an increase in the opposite bias, whereas we observe an increase in $\Delta f$ with significant bias in both bias polarities.

We thus consider the voltage $V$ being modified by a voltage drop caused by the tunneling current passing through the sample with resistivity $R_{s}$. Therefore $V=V_{\text {bias }}-I \cdot R_{s}$. The electrostatic force is then

$$
F_{t s}^{e s}=-\frac{1}{2} \frac{d C_{t s}}{d z}\left(V_{\text {bias }}^{2}-2 V_{\text {bias }} I R_{s}+I^{2} R_{s}^{2}\right)
$$

with an offset component proportional to $V_{\text {bias }}^{2}$, a term linear with $I$ and a quadratic term in $I$. At typical experimental conditions as in our previous experiments, where $V_{\text {bias }}=$ $1.5 \mathrm{~V}, R_{s}=150 \mathrm{M} \Omega$ and $I=1 \mathrm{nA},{ }^{3}$ the quadratic term is $5 \%$ of the linear term and can be neglected (however, for very small 
tip-sample distances as required for atomically resolved AFM on low-conductivity samples, this term cannot be neglected). Without the quadratic term, Eq. (3) reduces to

$$
F_{t s}^{e s} \approx-\frac{1}{2} \frac{d C_{t s}}{d z}\left(V_{\text {bias }}^{2}-2 V_{\text {bias }} I R_{s}\right) .
$$

In order to determine a relation between the frequency shift $\Delta f$ and the tunneling current $I$, we first have to calculate the contribution of this electrostatic force to the force gradient, $k_{t s}^{e s}$. After substituting $I=I_{0} e^{-\kappa z}$ into Eq. (4) and taking the derivative of $F_{t s}^{e s}$ with respect to $z$, Eq. (4) results in

$$
k_{t s}^{e s}=\frac{1}{2} \frac{d^{2} C_{t s}}{d z^{2}} V_{\text {bias }}^{2}-\left(\frac{d^{2} C_{t s}}{d z^{2}}-\frac{d C_{t s}}{d z} \kappa\right) V_{\text {bias }} I R_{s} .
$$

Since $\Delta f$ is directly proportional to $k_{t s}^{e s}$, assuming the small amplitude approximation, Eq. (5) can be rewritten as

$$
\Delta f=\frac{f_{0}}{4 k} \frac{d^{2} C_{t s}}{d z^{2}} V_{\text {bias }}^{2}-\frac{f_{0}}{2 k}\left(\frac{d^{2} C_{t s}}{d z^{2}}-\frac{d C_{t s}}{d z} \kappa\right) V_{\text {bias }} R_{s} I .
$$

The $\Delta f$ line shows a linear dependence with $I$ with an offset that depends on capacity and bias and a slope that is linear with $R_{s}$ and $V_{\text {bias. }}$. We define, from Eq. (6), the phantom force slope as

$$
\Xi:=\frac{d(\Delta f)}{d I}=-\frac{f_{0}}{2 k}\left(\frac{d^{2} C_{t s}}{d z^{2}}-\frac{d C_{t s}}{d z} \kappa\right) V_{\text {bias }} R_{s},
$$

which is usually expressed in $\frac{\mathrm{Hz}}{\mathrm{nA}}$. The slope is a measure of the strength of the phantom force.

\section{EXPERIMENTAL METHODS AND SETUP}

The experiments were performed in ultrahigh vaccum $\left(\approx 3 \times 10^{-10}\right.$ Torr $)$ and at room temperature. The images in this paper were all acquired in constant height mode. qPlus sensors $\left(k=1800 \frac{\mathrm{N}}{\mathrm{m}}\right)$ were equipped with tungsten tips to probe the sample. The tungsten tips were prepared by common techniques like controlled collision with the sample, field emission, and explosive delamination. ${ }^{23}$

The $\operatorname{Si}(111)-7 \times 7$ samples used were $p$ doped with $\rho=$ $6-9 \Omega \mathrm{cm}$ at $300 \mathrm{~K}$. The surface was prepared by repeated cycles of flashes up to $1250{ }^{\circ} \mathrm{C}$ followed by cooling periods in the range of several minutes.

The $n$-doped $\mathrm{Si}(100)$ samples had a resistivity of $0.008-$ $0.012 \Omega \mathrm{cm}$ at $300 \mathrm{~K}$. The surface was cleaned by the same flash routine as described above. After cleaning, approximately $300 \mathrm{~L}_{\text {deuterium }}{ }^{24}$ were deposited onto the surface at $\approx 450^{\circ} \mathrm{C}$.

\section{A. Investigation of a potential preamplifier artifact}

In the experimental setup, the bias voltage $V_{\text {bias }}$ was applied to the tip with the sample referenced to virtual ground via a preamplifier (preamp). The preamp is attached outside vacuum and amplifies, as a current-to-voltage converter, the $I$ signal by a factor of $10^{8} \frac{\mathrm{V}}{\mathrm{A}}$. Since the tip is oscillating, $I$ is an alternating current (ac) with a direct current (dc) offset, where only the dc component is measured by the preamp due to its limited bandwidth. To investigate if this phantom force effect is not due to a fluctuation of the virtual ground of the preamp, we introduced a switch that allows us to either connect the sample to real ground via a direct ground connection or the virtual (a)

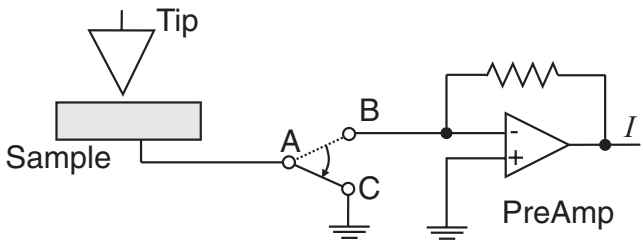

(b)

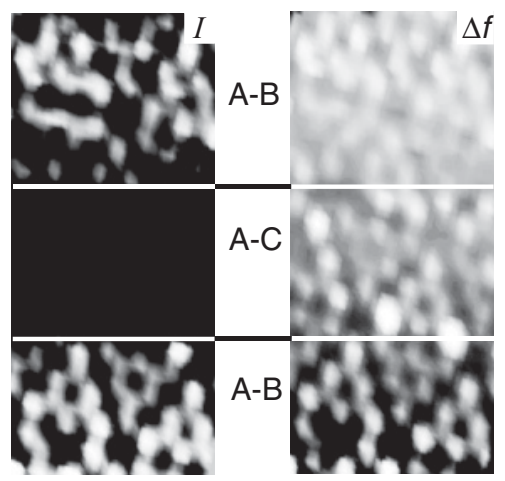

FIG. 3. (a) Schematic of the experimental setup. With an implemented switch, the preamp can be used for the $I$ signal (switch in position A-B) or can be shorted to ground (switch in position A-C). In (b), simultaneous acquired $I$ and $\Delta f$ data in constant height mode are shown. The images were scanned from top to bottom. In the middle section the $I$ signal was shorted to ground to check if the phantom force effect is real (i.e., caused by a voltage drop at the sample surface) or caused by a preamp artifact.

ground of the preamp, as schematically shown in Fig. 3(a). Because the operational amplifier used in the preamp has a limited gain, limited bandwidth, and a limited slew rate (in contrast to an ideal operational amplifier), the virtual ground terminal can deviate from zero, and cross coupling to the force gradient measurement might occur.

In the upper and lower section of Fig. 3(b), simultaneously recorded $I$ and $\Delta f$ data are presented in constant height mode (switch in position A-B). In the middle section of the image, the $I$ signal from the sample was shorted to ground (switch in position A-C). Nevertheless, the phantom force effect is still present in the $\Delta f$ signal, which clearly demonstrates that the phantom force is not caused by a preamp artifact, but by the current-induced local potential deviation outlined above.

\section{THE DEPENDENCE OF THE PHANTOM FORCE ON THE MACROSCOPIC TIP RADIUS ON SI(111)-7 ×7}

In the following section, we investigate the phantom force on the $\mathrm{Si}(111)-7 \times 7$ surface. If the metallic surface state does not play a measurable role, and the phantom force is highly localized, then the macroscopic tip radius $R$ [e.g., a large tip radius shown schematically in Fig. 4(a) versus a small tip radius shown in Fig. 4(b)] would not effect the phantom force. We would therefore not observe a dependence of the phantom force on the macroscopic tip radius $R$. We also discuss the results based on the description of the phantom force slope $\Xi$ given in Eq. (7).

Figures 5(a) and 5(b) show simultaneously acquired $I$ and $\Delta f$ data of the $\mathrm{Si}(111)-7 \times 7$ surface. In Fig. 5(a) the tunneling current reaches its maximum above the adatoms. In Fig. 5(b) 
(a)

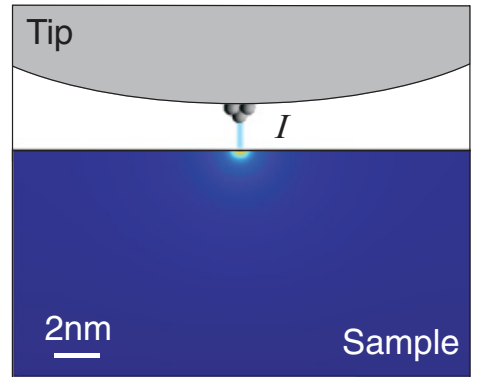

(b)

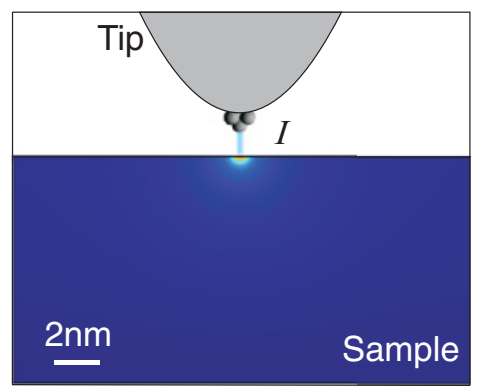

FIG. 4. (Color online) (a) Schematic of a tip with a large macroscopic radius in front of a surface with a highly localized change of the surface potential induced by the tunneling current. (b) The same situation, but with a smaller tip.

the brighter adatoms show a repulsive force contribution. The frequency shift is less negative over regions with a high tunneling current. A linear dependence between $\Delta f$ and the $I$ signal is shown in Fig. 5(c). By fitting the data we extracted a phantom force slope $\Xi=0.73 \frac{\mathrm{Hz}}{\mathrm{nA}}$.

The macroscopic tip can be described by its tip radius $R$, which we determined by fitting the long-range $\Delta f$ contribution between tip and sample to a model assuming only van der Waals interaction. ${ }^{25}$ In order to minimize the attractive
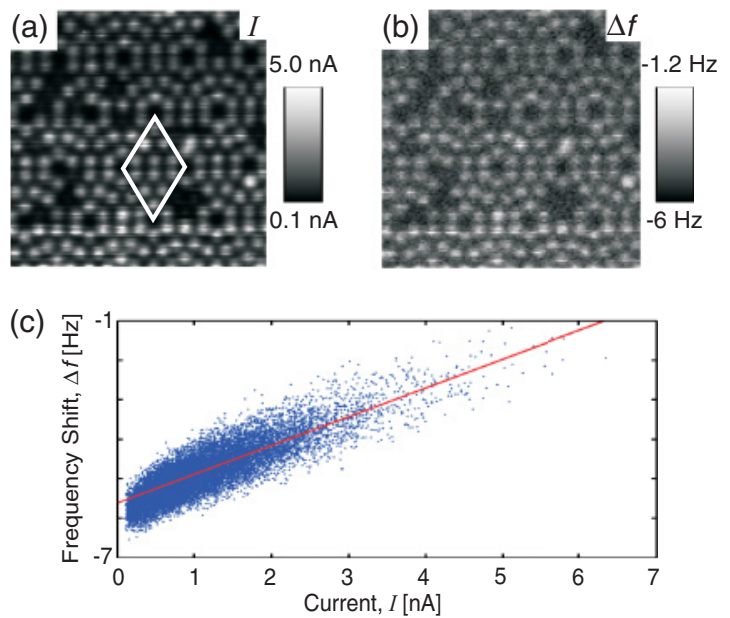

FIG. 5. (Color online) (a) Tunneling current $I$ and (b) $\Delta f$ data taken during constant height scanning. A $\operatorname{Si}(111)-7 \times 7$ unit cell is outlined by a white diamond in (a). In (b) the adatoms appear more bright, which is due to less attraction induced by the tunneling current. (c) Phantom force contribution as a linear dependence between the current and $\Delta f$ data. The phantom force slope $\Xi$ of the line is $0.73 \frac{\mathrm{Hz}}{\mathrm{nA}}$. Images are $10 \mathrm{~nm} \times 10 \mathrm{~nm}, A \approx 400 \mathrm{pm}, k=1800 \frac{\mathrm{N}}{\mathrm{m}}, f_{0}=19130 \mathrm{~Hz}$ and $V_{\text {bias }}=-1.5 \mathrm{~V}$. (a)

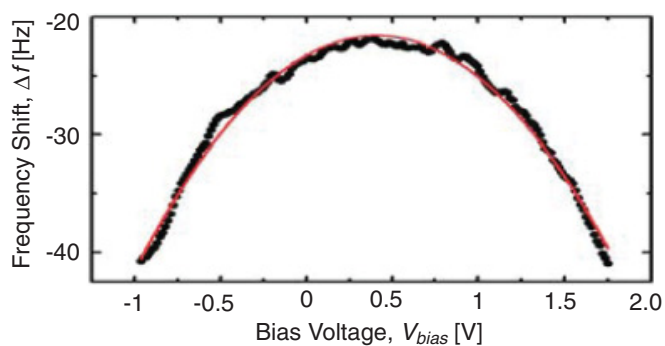

(b)

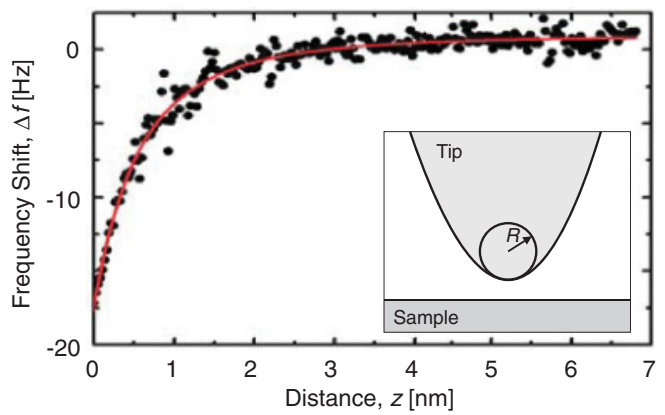

FIG. 6. (Color online) (a) Spectrum of $\Delta f\left(V_{\text {bias }}\right)$ for determining the $V_{\mathrm{CPD}}$ between tip and sample to $0.4 \mathrm{~V}$. (b) Spectrum of $\Delta f(z)$ taken at $V_{\mathrm{CPD}}$. The macroscopic tip radius $R$ was extracted by fitting the curve to a long-range van der Waals force contribution, for (b) the extracted $R=600 \mathrm{~nm}$. The inset shows the tip radius for a parabolic tip shape.

electrostatic force, we took $\Delta f(z)$ spectra while compensating for the $V_{\mathrm{CPD}}$. Before measuring the $\Delta f\left(V_{\text {bias }}\right)$, we retracted the tip from the sample by $100 \mathrm{pm}$. This reduced the possibility of tip-sample collisions due to drift. The voltage corresponding to the maximum $\Delta f$ value of the parabolic $\Delta f\left(V_{\text {bias }}\right)$ curve is $V_{\mathrm{CPD}} \cdot{ }^{26}$ In Fig. 6(a) the $V_{\mathrm{CPD}}$ was determined to be $0.4 \mathrm{~V}$. The $\Delta f(z)$ curves were fitted to a model incorporating a parabolic tip shape [as shown in Fig. 6(b)] in accordance to Refs. 25 and 27. The fit of the $\Delta f(z)$ data in Fig. 6(b) result in $R=600 \mathrm{~nm}$.

Figure 7 displays different phantom force slopes $\Xi$ dependent on the respective macroscopic tip radius $R$. Sixteen data points, acquired with two different qPlus sensors (sensor 1: red triangles; sensor 2: blue dots), are plotted. The data points are widely spread and range from radii of 51-6775 nm.

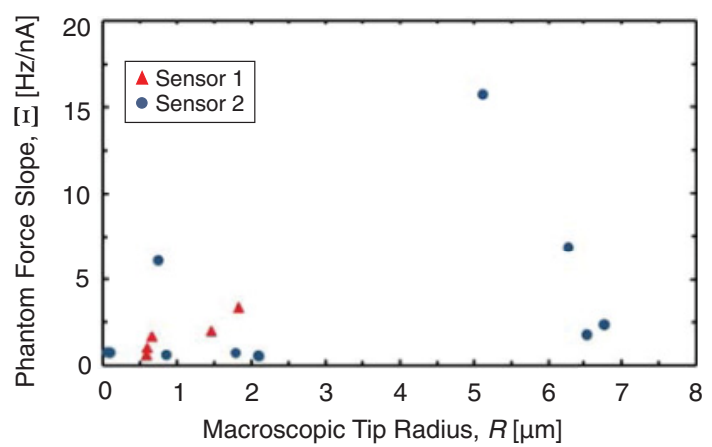

FIG. 7. (Color online) The phantom force slope $\Xi$ in $\frac{\mathrm{Hz}}{\mathrm{nA}}$ characterizing the phantom force is plotted versus the macroscopic tip radii $R$. Two different sensors were used: sensor 1 (red triangles) and sensor 2 (blue dots). The phantom force slopes $\Xi$ show no dependence on the macroscopic tip radii $R$. 
The phantom force slopes vary from $0.51 \frac{\mathrm{Hz}}{\mathrm{nA}}$ to $15.74 \frac{\mathrm{Hz}}{\mathrm{nA}}$. In particular, slopes below $2.0 \frac{\mathrm{Hz}}{\mathrm{nA}}$ can be observed for a wide range of macroscopic tip radii $R$. We observe no dependence between the phantom force slope and the macroscopic tip radius. This supports our hypothesis of a highly local voltage drop, and suggests that the metallic surface state does not play a role.

The spread in our measurements of Fig. 7 can be discussed with the aid of Eq. (7), $\Xi=-\frac{f_{0}}{2 k}\left(\frac{d^{2} C_{t s}}{d z^{2}}-\frac{d C_{t s}}{d z} \kappa\right) V_{\text {bias }} R_{s}$. We turn now to the factors $-\frac{f_{0}}{2 k}\left(\frac{d^{2} C_{t s}}{d z^{2}}-\frac{d C_{t s}}{d z} \kappa\right)$ and $R_{s}$ in detail, as $V_{\text {bias }}$ was constant for these measurements.

The factor $-\frac{f_{0}}{2 k}\left(\frac{d^{2} C_{t s}}{d z^{2}}-\frac{d C_{t s}}{d z} \kappa\right)$ can be calculated, assuming a model of the electrostatic force $F_{t s}^{e s}$ of a conical tip (half angle $\theta$ ) in front of a metallic surface as described by Hudlet et al. ${ }^{16}$ This would be applicable, if the tip and sample surfaces could be modelled by a constant potential. Calculations for realistic $R=5 \mathrm{~nm}$ and $\theta=70^{\circ}$, at conditions summarized in Ref. 28, lead to unrealistic values of $\Xi=68 \frac{\mathrm{Hz}}{\mathrm{nA}}$, much larger than $2.8 \frac{\mathrm{Hz}}{\mathrm{nA}}$, the experimentally determined average $\Xi$ of the values shown in Fig. 7 . We propose that this phantom force effect is highly localized. Instead of being described by the macroscopic tip shape, it would be better described by a model of the nanoscopic tip cluster. Assuming a plate capacitor with $C=\epsilon_{0} \frac{A}{z}$, we can calculate the phantom force slope using Eq. (7) and the following parameters: $f_{0}=20 \mathrm{kHz}$, $k=1800 \mathrm{~N} / \mathrm{m}, \kappa=2 \AA^{-1}, R_{s}=150 \mathrm{M} \Omega$ with an applied bias $V_{\text {bias }}=-1.5 \mathrm{~V}$, at a distance $z=4.4 \AA$ and a capacitive area $A=(1 \mathrm{~nm})^{2}$. This yields a slope $\Xi=2.8 \frac{\mathrm{Hz}}{\mathrm{nA}}$, equivalent to the experimental average of $2.8 \frac{\mathrm{Hz}}{\mathrm{nA}}$. While a parallel plate capacitor might be an oversimplification at this length scale, local capacitive interaction (that is, a parabolic dependence of the force with respect to an applied bias voltage) between tip and sample has been reported at the atomic scale. ${ }^{29}$

Concerning the factor $R_{s}$, we observed in Ref. 3 that the higher the sample resistivity the higher the slope $\Xi$. In our case, the data points with higher $\Xi$ were collected on areas with an increased number of defects on the $\mathrm{Si}(111)-7 \times 7$ surface. The dependence between $\Xi$ and the defect density on the $\mathrm{Si}(111)-7 \times 7$ surface was investigated and is shown in Fig. 8 . For low defect densities, $\Xi$ seems to be low in contrast to higher defect densities with an increased $\Xi$. But, since the tip shape and $R_{s}$ changed for each data point, the dependence

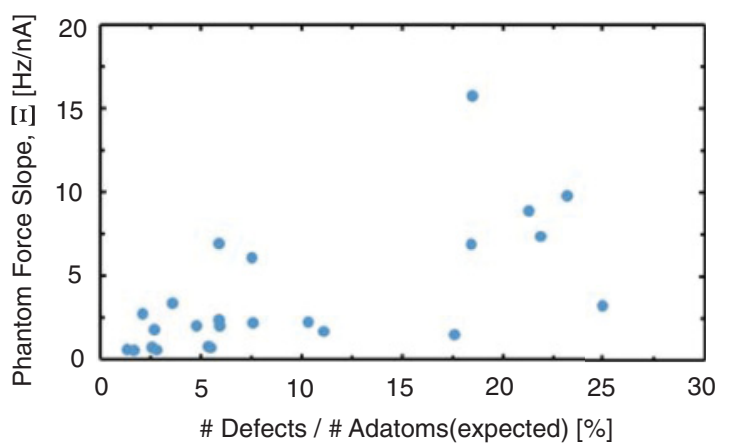

FIG. 8. (Color online) Phantom force slopes $\Xi$ plotted versus the defect density on the $\mathrm{Si}(111)-7 \times 7$ surface. The phantom force slopes $\Xi$ seem small for less defects on the $\operatorname{Si}(111)-7 \times 7$ and more defects point to increased $\Xi$. between phantom force slopes and the defect densities is not conclusive and has to be investigated in more detail.

\section{THE PHANTOM FORCE ON THE HYDROGENATED SI(100) SURFACE}

In this section, we present measurements on the $\mathrm{H} / \mathrm{Si}(100)$ surface. Exposing $\mathrm{Si}(100)$ to hydrogen saturates the unsaturated dangling bonds. ${ }^{30}$ The electronic states of the hydrogenated dimers have been shown to be outside the band gap of bulk $\mathrm{Si}$, meaning that in contrast to $\mathrm{Si}(111)-7 \times 7$, the surface does not have a metallic surface state. ${ }^{31}$

In Fig. 9(a), a tunneling current between tip and sample, which leads to the phantom force effect, is schematized. Figures 9(b) and 9(c) show simultaneous $\Delta f$ and $I$ data collected at constant height with an applied bias voltage of $1.5 \mathrm{~V}$. The dimer rows can be seen running from upper left to lower right. The low contrast is due to our choice of a relatively large

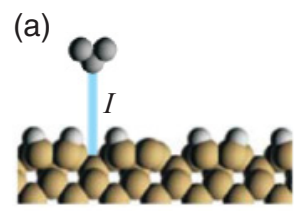

(e)
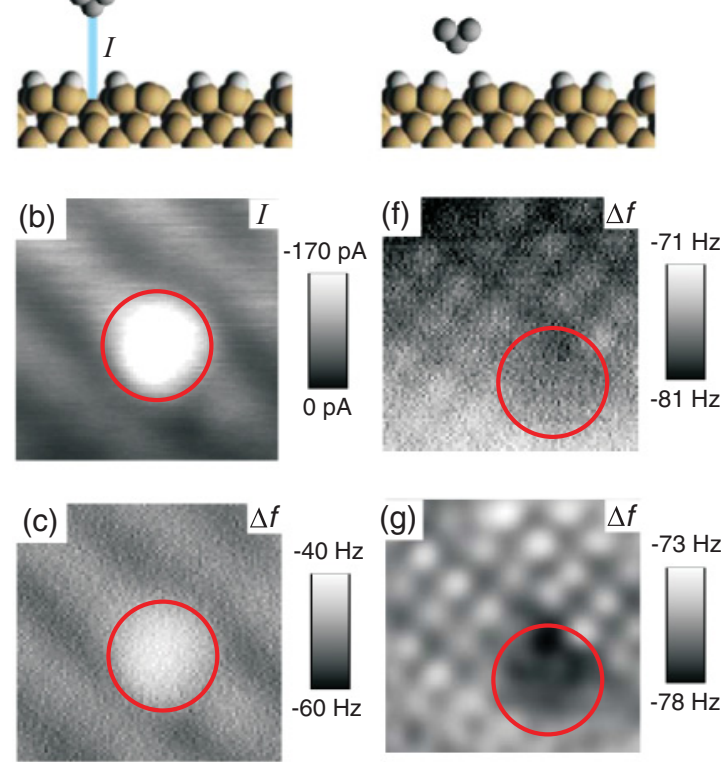

(d)

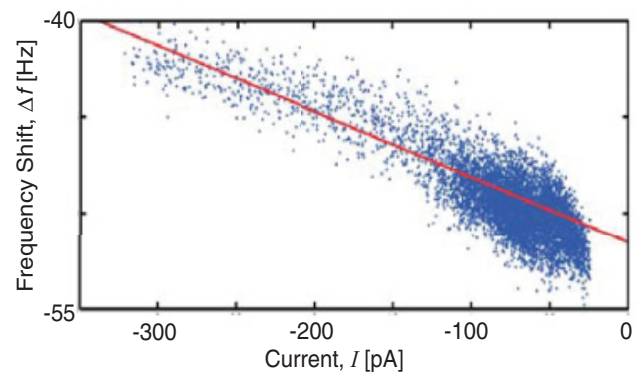

FIG. 9. (Color online) (a) At tip-sample distances characteristic for STM and with a tunneling current, the phantom force effect can be observed. Simultaneous acquired $I$ and $\Delta f$ data with atomic contrast are shown in (b) and (c) for $V_{\text {bias }}=1.5 \mathrm{~V}$. In (d), the relation between $\Delta f$ and $I$ data is plotted. Data points with increased $I$ data, as the defect outlined in red, show a stronger decrease (less attraction) of the $\Delta f$ values. However, at close tip-sample distances, (e), and low bias, in this case $200 \mathrm{mV}$, the defect appears darker (more attractive), (f). Image (g) shows (f) with low-pass filtering and plane substraction applied for clarity. Images are $2 \mathrm{~nm} \times 2 \mathrm{~nm}, A=$ $100 \mathrm{pm}, k=1800 \frac{\mathrm{N}}{\mathrm{m}}, f_{0}=19131 \mathrm{~Hz}$. 
imaging distance to prevent excessive tunneling currents when scanning over the defect area, circled in red. The feature circled in red is most likely a dangling bond, which we would expect to observe in $\Delta f$ data as darker (more attractive). However, due to the increase of the tunneling current over it, the phantom force effect causes an increase in $\Delta f$ that makes it appear brighter. To investigate the relationship between $\Delta f$ and $I$, we plotted in Fig. 9(d) the $\Delta f$ information of each single pixel in image (c) versus the corresponding pixel of the $I$ information in (b). For positive applied bias voltages, the $I$ signal is negative. The relation between $\Delta f$ and $I$ data results in $\Xi=-34 \frac{\mathrm{Hz}}{\mathrm{nA}}$, if we assume a linear relation as described in Eq. (6).

In Fig. 9(e), the bias voltage is decreased to $200 \mathrm{mV}$ and in order to resolve atomic contrast, the tip must be approached to the surface, similar to our previous observations of the phantom force. ${ }^{3}$ The attractive interaction in the presence of the dangling bond is clearly observed in $\Delta f$ data collected at low bias, as shown in Fig. 9(f). Figure 9(g) is a low-pass filtered and plane substracted image from (f) to show the dangling bond with better contrast. We demonstrated that the phantom force does not depend on the presence of a metallic surface state and still appears on a sample system as $\mathrm{H} / \mathrm{Si}(100)$ without a metallic surface state.

It is useful to contrast the phantom force with a phenomenon observed on semiconductors without a metallic surface state, known as tip-induced band bending (TIBB). Without a metallic surface, the electric field of the tip penetrates into the surface and part of the voltage between tip and sample drops in the sample itself. ${ }^{32}$ In contrast to the phantom force, TIBB can have a lateral extension in the range of tens of $\mathrm{nm}$ and is strongly dependent on the macroscopic tip radius. ${ }^{33}$ TIBB does not require a current; it is a voltage drop within the sample that itself changes the tunneling current. The phantom force, on the other hand, is a current-induced drop of voltage within the sample. These two phenomena manifest themselves differently upon the attractive electrostatic force: the voltage drop induced by TIBB is not current dependent and the attractive electrostatic force should be constant in a small scan area, whereas the phantom force can explain atomic-scale variations in the electrostatic force that vary with current. It should be noted as well that STM investigations of TIBB also indicated current-related effects, possibly related to the phantom force. ${ }^{34}$

\section{SUMMARY AND OUTLOOK}

Section III introduces to the equipment and methods used for the experiments. It was shown that the phantom force is not an effect of the tunneling current amplifier.

In Sec. IV, we investigated the influence of a metallic surface state on the phantom force. The experimental observation of the phantom force slope $\Xi$ shows no dependence on the macroscopic tip radius $R$. This infers a highly localized voltage drop and we concluded that the metallic surface state does not play a role in the phantom force effect. Finally, in Sec. V, we showed that the phantom force is present on a sample system without a metallic surface state, which had not explicitly been demonstrated experimentally.

For a future project we suggest low-temperature measurements to investigate the dependence of the phantom force on the defect density on $\operatorname{Si}(111)-7 \times 7$. In this experiment, the tip would be more stable and a controlled exposure of a distinct spot on the surface to, e.g., oxygen could clarify the dependence between phantom force and sample resistivity.

\section{ACKNOWLEDGMENTS}

The authors thank the German Science Foundation (DFG, Sonderforschungsbereich 689) for financial support, and J. Welker, M. Emmrich, E. Wutscher, and F. Pielmeier for helpful discussions. *jay.weymouth@physik.uni-r.de

${ }^{1}$ M. Ternes, C. Gonzalez, C. P. Lutz, P. Hapala, F. J. Giessibl, P. Jelinek, and A. J. Heinrich, Phys. Rev. Lett. 106, 016802 (2011).

${ }^{2}$ Y. Sun, H. Mortensen, S. Schär, A. S. Lucier, Y. Miyahara, P. Grütter, and W. Hofer, Phys. Rev. B 71, 193407 (2005).

${ }^{3}$ A. J. Weymouth, T. Wutscher, J. Welker, T. Hofmann, and F. J. Giessibl, Phys. Rev. Lett. 106, 226801 (2011).

${ }^{4}$ K. Takayanagi, Y. Tanishiro, and S. Takahashi, J. Vac. Sci. Technol. A 3, 1502 (1985).

${ }^{5}$ M. Mauerer, I. L. Shumay, W. Berthold, and U. Höfer, Phys. Rev. B 73, 245305 (2006).

${ }^{6}$ B. N. J. Persson, Phys. Rev. B 34, 5916 (1986).

${ }^{7}$ S. Hasegawa and S. Ino, Phys. Rev. Lett. 68, 1192 (1992).

${ }^{8}$ Y. Hasegawa, I.-W. Lyo, and Ph. Avouris, Appl. Surf. Sci. 76-77, 347 (1994).

${ }^{9}$ S. Heike, S. Watanabe, Y. Wada, and T. Hashizume, Phys. Rev. Lett. 81, 890 (1998).

${ }^{10}$ M. D'angelo, K. Takase, N. Miyata, T. Hirahara, S. Hasegawa, A. Nishide, M. Ogawa, and I. Matsuda, Phys. Rev. B 79, 035318 (2009).

${ }^{11}$ T. Tanikawa, K. Yoo, I. Matsuda, S. Hasegawa, and Y. Hasegawa, Phys. Rev. B 68, 113303 (2003).
${ }^{12}$ K. Yoo and H. H. Weitering, Phys. Rev. B 65, 115424 (2002).

${ }^{13}$ J. W. Wells, J. F. Kallehauge, T. M. Hansen, and Ph. Hofmann, Phys. Rev. Lett. 97, 206803 (2006).

${ }^{14}$ [http://www.comsol.com], version 4.2 (2011).

${ }^{15}$ L. Olsson, N. Lin, V. Yakimov, and R. Erlandsson, J. Appl. Phys. 84, 8 (1998).

${ }^{16} \mathrm{~S}$. Hudlet, M. Saint-Jean, C. Guthmann, and J. Berger, Eur. Phys. J. B 25, 5 (1998).

${ }^{17}$ T. R. Albrecht, P. Grütter, D. Horne, and D. Rugar, J. Appl. Phys. 69, 668 (1991).

${ }^{18}$ F. J. Giessibl, Rev. Mod. Phys. 75, 949 (2003).

${ }^{19}$ F. J. Giessibl, Appl. Phys. Lett. 78, 123 (2001).

${ }^{20} \mathrm{G}$. Binnig, H. Rohrer, Ch. Gerber, and E. Weibel, Phys. Rev. Lett. 50, 120 (1983).

${ }^{21}$ M. Nonnenmacher, M. P. O’Boyle, and H. K. Wickramasinghe, Appl. Phys. Lett. 58, 2921 (1991).

${ }^{22}$ S. Sadewasser, P. Jelinek, C. K. Fang, O. Custance, Y. Yamada, Y. Sugimoto, M. Abe, and S. Morita, Phys. Rev. Lett. 103, 266103 (2009).

${ }^{23}$ T. Hofmann, J. Welker, and F. J. Giessibl, J. Vac. Sci. Technol. B 28, C4E28 (2010).

${ }^{24}$ The $\mathrm{Si}(100)$ surface was saturated with deuterium, which for purposes of this study behaves as hydrogen. 
${ }^{25}$ H. Hölscher, U. D. Schwarz, and R. Wiesendanger, Appl. Surf. Sci. 140, 344 (1999).

${ }^{26}$ M. Guggisberg, M. Bammerlin, Ch. Loppacher, O. Pfeiffer, A. Abdurixit, V. Barwich, R. Bennewitz, A. Baratoff, E. Meyer, and H.-J. Güntherodt, Phys. Rev. B 61, 11151 (2000).

${ }^{27}$ F. J. Giessibl, Phys. Rev. B 56, 16010 (1997).

${ }^{28}$ Further conditions for the calculation of $\Xi$ were: $f_{0}=20000 \mathrm{~Hz}$, $k=1800 \frac{\mathrm{N}}{\mathrm{m}}, \kappa=2 \AA^{-1}, L_{\text {tip }}=300 \mu \mathrm{m}, z=500 \mathrm{pm}, V_{\text {bias }}=-1.5$ $\mathrm{V}, R_{s}=150 \mathrm{M} \Omega$.
${ }^{29}$ L. Gross, F. Mohn, P. Liljeroth, J. Repp, F. J. Giessibl, and G. Meyer, Science 324, 1428 (2009).

${ }^{30}$ J. J. Boland, Phys. Rev. Lett. 65, 3325 (1990).

${ }^{31}$ H. Raza, Phys. Rev. B 76, 045308 (2007).

${ }^{32}$ R. M. Feenstra, Y. Dong, M. P. Semtsiv, and W. T. Masselink, Nanotechnology 18, 044015 (2007).

${ }^{33}$ R. M. Feenstra, J. Vac. Sci. Technol. B 21, 2080 (2003).

${ }^{34}$ R. M. Feenstra, G. Meyer, and K. H. Rieder, Phys. Rev. B 69, 081309 (2004). 